\title{
Peroxiredoxin isoforms are associated with cardiovascular risk factors in type 2 diabetes mellitus
}

\author{
E. El Eter ${ }^{1,2,3}$ and A.A. Al-Masri ${ }^{1,2}$ \\ ${ }^{1}$ Physiology Department, Faculty of Medicine, King Saud University, Riyadh, Saudi Arabia \\ ${ }^{2}$ Cardiovascular Research Group, Faculty of Medicine, King Saud University, Riyadh, Saudi Arabia \\ ${ }^{3}$ Physiology Department, Faculty of Medicine, Alexandria University, Alexandria, Egypt
}

\begin{abstract}
The production of oxygen free radicals in type 2 diabetes mellitus contributes to the development of complications, especially the cardiovascular-related ones. Peroxiredoxins (PRDXs) are antioxidant enzymes that combat oxidative stress. The aim of this study was to investigate the associations between the levels of PRDX isoforms (1, 2, 4, and 6) and cardiovascular risk factors in type 2 diabetes mellitus. Fifty-three patients with type 2 diabetes mellitus $(28 \mathrm{~F} / 25 \mathrm{M})$ and 25 healthy control subjects $(7 \mathrm{~F} / 18 \mathrm{M})$ were enrolled. We measured the plasma levels of each PRDX isoform and analyzed their correlations with cardiovascular risk factors. The plasma PRDX1, -2, -4, and -6 levels were higher in the diabetic patients than in the healthy control subjects. PRDX2 and -6 levels were negatively correlated with diastolic blood pressure, fasting blood sugar, and hemoglobin A1c. In contrast, PRDX1 levels were positively correlated with low-density lipoprotein and C-reactive protein levels. PRDX4 levels were negatively correlated with triglycerides. In conclusion, PRDX1, -2, -4, and -6 showed differential correlations with a variety of traditional cardiovascular risk factors. These results should encourage further research into the crosstalk between PRDX isoforms and cardiovascular risk factors.
\end{abstract}

Key words: Peroxiredoxins; Type 2 diabetes mellitus; Oxidative stress; Cardiovascular risk factors

\section{Introduction}

Oxidative stress is known to play a role in various diseases, including diabetes mellitus and its cardiovascular complications. Oxidative stress usually results from an imbalance between the production of reactive oxygen species (ROS) and/or reduced antioxidant capacity (1). There is growing evidence for a link between free radical production and oxidative stress in the pathogenesis of cardiovascular disease (2) because inflammation increases endothelial cell damage and microvascular permeability (3). Cardiovascular disease is a leading cause of death worldwide. Therefore, it is essential to identify patients with diabetes at high risk of cardiovascular events (4). Although there are abundant clinical data showing associations between markers of oxidative stress and common cardiovascular risk factors, there are limited data linking peroxiredoxins (PRDXs) with cardiovascular risk factors.

Cells are supported by protective antioxidant systems, which reduce the concentrations of ROS in the intracellular and extracellular environments. PRDXs are a group of antioxidant proteins containing an essential catalytic cysteine that use thioredoxin to scavenge hydrogen peroxide, lipid hydroperoxides, and peroxynitrite (5). They provide a potent defense mechanism that maintains the redox balance in normal and oxidative stress conditions. In addition, they possess a chaperone activity (6). Six isoforms (PRDX1-6) have been identified and they are expressed in sites of ROS production, including the cytosol, mitochondria, and peroxisomes (5).

The lower antioxidant capacity in diabetic patients is due to reduced activity of antioxidant enzymes, including superoxide dismutase, catalase, and glutathione peroxidase $(7,8)$. Although an association between PRDX expression and diabetes is already known, the association between this family of enzymes and cardiovascular risk factors is still unknown. Therefore, the present study was designed to determine the baseline levels of circulating PRDX isoforms $(1,2,4$, and 6) and their correlations with traditional cardiovascular risk factors, including blood pressure (BP), hyperlipidemia, and C-reactive protein (CRP) in patients with type 2 diabetes mellitus (T2DM). 


\section{Materials and Methods}

\section{Study population}

In the present study, 53 patients with T2DM were recruited at the primary care clinic at King Khalid University Hospital (KKUH), Riyadh, Saudi Arabia. Exclusion criteria included acute onset of clinical or laboratory signs of acute infection, myocardial infarction, stroke, trauma, or surgical procedure in the last 6 months. Patients with coexisting malignant tumor, hepatic disease, end stage renal disease (dialysis), immunosuppression, or the presence of complications such as neuropathy, nephropathy and retinopathy were also excluded. The full medical records of the patients were retrieved, and included detailed physical findings and routine clinical laboratory tests. The duration of diabetes ranged from 7 to 10 years, and all of the patients had non-insulin-dependent diabetes mellitus. All of the patients were receiving anti-diabetic and anti-hypertensive agents. The control group comprised 25 non-diabetic subjects. The medical history was assessed and physical examinations were performed in all subjects. The study was carried out in accordance with the institutional review board regulations of the Medical College at King Saud University. The study protocol was approved by the College of Medicine and University Hospital Ethics Committee, and written informed consent was obtained from all participants.

\section{Blood samples and determination of PRDX levels}

Blood samples were taken after an overnight fast of 10 $12 \mathrm{~h}$ from the antecubital vein and were collected in native, ethylenediaminetetraacetic acid (EDTA)-coated tubes. The samples were centrifuged at $5000 \mathrm{~g}$ for $10 \mathrm{~min}$ and aliquots of serum and plasma were stored at $-70^{\circ} \mathrm{C}$ until assayed. PRDX1, $-2,-4$, and -6 levels were measured in plasma samples from patients and healthy controls using commercially available enzyme-linked immunosorbent assay (ELISA) kits from Wuhan EIAab Science Co., Ltd. (China) or Abfrontier for PDRX1 (China) according to the manufacturers' instructions. Fasting serum insulin levels were determined in plasma samples using ELISA kits from R\&D Systems (USA) according to the manufacturer's instructions.

Fasting blood sugar (FBS) was analyzed at KKUH Biochemistry Central Laboratory using a Konelab Intelligent Diagnostics Systems analyzer (Konelab Corporation, Finland). Glycated hemoglobin (HbA1c) was measured using a Helena Glyco-Tek Affinity Column (Helena Biosciences, UK). Homeostaisis model assessment of insulin resistance (HOMA-IR) was calculated according to the following equation: fasting insulin $(\mu \mathrm{U} / \mathrm{L}) \times$ fasting glucose $(n M) / 22.5$.

\section{Statistical analysis}

Data entry and statistical analysis were performed using SPSS statistical software, version 18 (USA). Error bars were used to compare the mean and $95 \%$ confidence intervals (Cl) of the plasma PRDX levels between the two groups.
Pearson's correlation coefficient was used to determine correlations between PRDX levels and other variables, and $x-y$ scatterplots were plotted. Normally distributed values are reported as means $\pm S D$. Student's $t$-test was used to compare continuous variables and the $\chi^{2}$ test was used to compare categorical variables between the two groups. In all analyses, a $P$ value of $<0.05$ was set as the level of significance.

\section{Results}

\section{Clinical characteristics of study subjects}

Table 1 compares the biochemical and clinical characteristics between the control nondiabetic subjects and diabetic subjects. The diabetic patients had metabolic disturbances, including elevated levels of FBS, HbA1c, serum triglycerides (TG), and low-density lipoproteins (LDL) compared with the control subjects. Systolic blood pressure (SBP) was greater in diabetic subjects than in control subjects, despite the higher use of anti-hypertensive drugs in the diabetic subjects.

\section{PRDXs levels in diabetic patients}

Table 2 shows the plasma levels of the four PRDX isoforms in the healthy control subjects and diabetic subjects. PRDX1, -2, -4 and -6 levels were greater in diabetic subjects than in control subjects.

\section{Correlations between plasma PRDX levels and cardiovascular risk factors}

We examined whether the plasma PRDX levels were

Table 1. Clinical characteristics of the non-diabetic healthy control subjects and diabetic patients.

\begin{tabular}{lcc}
\hline & $\begin{array}{c}\text { Non-diabetic } \\
\text { subjects }(n=25)\end{array}$ & $\begin{array}{c}\text { Diabetic } \\
\text { patients }(n=53)\end{array}$ \\
\hline Age & $54.15 \pm 15.39$ & $61.33 \pm 11.14^{*}$ \\
Gender (F/M) & $8 / 17$ & $28 / 25$ \\
BMI & $25.26 \pm 4.06$ & $26.79 \pm 4.32$ \\
SBP (mmHg) & $117.56 \pm 3.9$ & $135.59 \pm 21.95^{*}$ \\
DBP (mmHg) & $75.89 \pm 6.277$ & $75.85 \pm 9.06$ \\
Serum insulin (IU/mL) & $13.17 \pm 5.86$ & $17.75 \pm 14.94$ \\
FBS (mg/dL) & $4.71 \pm 0.544$ & $9.67 \pm 4.09^{*}$ \\
HOMA-IR & $2.82 \pm 1.437$ & $7.14 \pm 6.2^{*}$ \\
Serum cholesterol (mM) & $4.49 \pm 0.617$ & $4.76 \pm 1.38$ \\
Serum TG (mM) & $0.92 \pm 0.39$ & $1.86 \pm 1.07^{*}$ \\
HDL (mM) & $0.99 \pm 0.314$ & $1.17 \pm 1.16$ \\
LDL (mM) & $2.7 \pm 0.657$ & $3.64 \pm 1.45^{*}$ \\
\hline
\end{tabular}

Data are reported as means \pm SD. BMI: body mass index; SBP: systolic blood pressure; DBP: diastolic blood pressure; FBS: fasting blood sugar; HOMA-IR: homeostaisis model assessment of insulin resistance; TG: triglycerides; HDL: high-density lipoprotein; LDL: low-density lipoprotein. ${ }^{*} \mathrm{P}<0.05$ compared to non-diabetic control subjects (unpaired Student's $t$-test). 
Table 2. Plasma levels of peroxiredoxin isoforms in non-diabeitc healthy control subjects and diabetic patients

\begin{tabular}{lcc}
\hline & $\begin{array}{c}\text { Non-diabetic } \\
\text { subjects }(\mathrm{n}=25)\end{array}$ & $\begin{array}{c}\text { Diabetic } \\
\text { patients }(\mathrm{n}=53)\end{array}$ \\
\hline PRDX1 $(\mathrm{ng} / \mathrm{mL})$ & $16.77 \pm 3.899$ & $21.92 \pm 5.77^{*}$ \\
PRDX2 $(\mathrm{ng} / \mathrm{mL})$ & $20.37 \pm 8.61$ & $36.61 \pm 14.966^{*}$ \\
PRDX4 $(\mathrm{pg} / \mathrm{mL})$ & $2696 \pm 1972$ & $3835 \pm 1454^{*}$ \\
PRDX6 $(\mathrm{pg} / \mathrm{mL})$ & $238 \pm 111$ & $311 \pm 111^{*}$ \\
\hline
\end{tabular}

Data are reported as means \pm SD. PRDX: peroxiredoxin. ${ }^{*} \mathrm{P}<0.05$ compared to non-diabetic control subjects (unpaired Student's $t$-test).

correlated with cardiovascular risk factors, including SBP, diastolic blood pressure (DBP), FBS, HbA1c, CRP, TG, LDL, and cholesterol. Table 3 shows the correlations between

Table 3. Pearson correlations of plasma levels of peroxiredoxin isoforms and other measured variables of all diabetic patients $(n=53)$.

\begin{tabular}{|c|c|c|c|c|c|}
\hline & & PRDX1 & PRDX2 & PRDX4 & PRDX6 \\
\hline \multirow[t]{2}{*}{ Weight (kg) } & $r$ & 0.071 & -0.081 & 0.109 & -0.057 \\
\hline & $P$ & 0.640 & 0.591 & 0.469 & 0.706 \\
\hline \multirow[t]{2}{*}{ Height (cm) } & $r$ & 0.025 & -0.141 & -0.006 & -0.107 \\
\hline & $P$ & 0.872 & 0.360 & 0.970 & 0.490 \\
\hline \multirow[t]{2}{*}{$\mathrm{SBP}(\mathrm{mmHg})$} & $r$ & -0.051 & -0.213 & -0.222 & -0.198 \\
\hline & $P$ & 0.728 & 0.142 & 0.126 & 0.172 \\
\hline \multirow[t]{2}{*}{ DBP (mmHg) } & $r$ & -0.052 & $-0.380^{* *}$ & -0.261 & $-0.388^{* *}$ \\
\hline & $P$ & 0.722 & 0.007 & 0.070 & 0.006 \\
\hline \multirow[t]{2}{*}{$\mathrm{ESR}(\mathrm{mm})$} & $r$ & $0.474^{* *}$ & 0.149 & 0.166 & 0.070 \\
\hline & $P$ & 0.001 & 0.301 & 0.249 & 0.631 \\
\hline \multirow[t]{2}{*}{ FBS (mM) } & $r$ & 0.031 & $-0.308^{*}$ & -0.138 & $-0.281^{*}$ \\
\hline & $P$ & 0.831 & 0.030 & 0.341 & 0.048 \\
\hline \multirow[t]{2}{*}{$\mathrm{HbA} 1 \mathrm{c}$} & $r$ & 0.120 & $-0.303^{*}$ & -0.102 & $-0.309^{*}$ \\
\hline & $P$ & 0.405 & 0.033 & 0.481 & 0.029 \\
\hline \multirow[t]{2}{*}{ CRP } & $r$ & $0.429^{* *}$ & 0.227 & 0.131 & 0.158 \\
\hline & $P$ & 0.004 & 0.144 & 0.401 & 0.312 \\
\hline \multirow[t]{2}{*}{ TC (mM) } & $r$ & 0.082 & -0.190 & -0.028 & -0.103 \\
\hline & $P$ & 0.579 & 0.197 & 0.851 & 0.487 \\
\hline \multirow[t]{2}{*}{ TG (mM) } & $r$ & 0.142 & -0.276 & $-0.481^{* *}$ & -0.169 \\
\hline & $P$ & 0.331 & 0.055 & 0.000 & 0.247 \\
\hline \multirow[t]{2}{*}{ HDL (mM) } & $r$ & -0.436 & 0.355 & $0.666^{*}$ & 0.452 \\
\hline & $P$ & 0.208 & 0.315 & 0.035 & 0.190 \\
\hline \multirow[t]{2}{*}{ LDL (mM) } & $r$ & $0.813^{\star *}$ & -0.484 & -0.193 & -0.512 \\
\hline & $P$ & 0.004 & 0.156 & 0.594 & 0.130 \\
\hline
\end{tabular}

Data are reported as Pearson correlation coefficients and twosided P-values. PRDX: peroxiredoxin; SBP: systolic blood pressure; $r$. Pearson correlation coefficient; DBP: diastolic blood pressure; ESR: erythrocyte sedimentation rate; FBS: fasting blood sugar level; HbA1c: glycated hemoglobin; CRP: C-reactive protein; TC: total cholesterol; TG: triglycerides; HDL: high-density lipoprotein; LDL: low-density lipoprotein. ${ }^{*} \mathrm{P}<0.05$, ${ }^{* *} \mathrm{P}<0.01$ are considered to be statistically significant these variables and the circulating PRDX levels. The results suggest that the individual PRDX isoforms were differentially associated with the cardiovascular risk factors determined in this study.

\section{Discussion}

The present study revealed that the plasma levels of PRDX isoforms (PRDX1, -2, -4, and -6), antioxidant enzymes, were greater in diabetic patients compared with healthy subjects. Our data also revealed differential associations between the individual PRDX isoforms and cardiovascular risk factors. In particular, PRDX2 and -6 levels were negatively correlated with DBP, FBS, and HbA1c. In contrast, PRDX1 levels were positively correlated with LDL and CRP levels, while PRDX4 levels were negatively correlated with TG levels.

Prior studies have shown that oxidative stress, which is characterized by increased production of ROS and/or decreased antioxidant defense mechanisms, plays an important role in the pathogenesis of diabetes (7) and its complications (9). In the present study, we focused on four members of the PRDX family and PRDX1, -2, -4, and -6 were markedly elevated in T2DM patients compared with control subjects. Because there are currently no data regarding the normal range of circulating PRDXs, except for previously published results from our laboratory $(10,11)$, we assumed that the levels in the healthy control subjects in this study are not pathological. We made this assumption because we applied strict selection criteria for the control subjects and diabetic subjects. Previous studies in patients with T2DM revealed a decrease in antioxidant defense mechanisms and an increase in markers of oxidative damage, especially in patients with diabetes-related complications (9). Similar results to ours were reported for PRDX1 activity in erythrocytes, which was greater in T2DM patients than in non-diabetic subjects (12). Another study revealed a high total antioxidant capacity and higher concentrations of lipid peroxidation markers in T2DM patients than in non-diabetic subjects (13). We observed higher antioxidant levels in the present study, despite the well-known increase in oxidative stress in diabetes. These results suggest a possible adaptive response that is probably due to the increased production of $\mathrm{O}_{2}^{-}$, which would lead to augmented $\mathrm{H}_{2} \mathrm{O}_{2}$ production. This mechanism may require a higher activity of antioxidant enzymes to protect against the increased level of oxidative stress in relation to adverse cardiovascular and metabolic conditions.

BP control was strongly correlated with cardiovascular disease in studies investigating cardiovascular risk factors in diabetes. Additionally, an improvement in BP control was reported to significantly reduce the risk of cardiovascular disease in diabetic patients $(14,15)$. SBP $>140 \mathrm{mmHg}$ was associated with an increased risk for cardiovascular disease in T2DM patients (16). In our study, SBP was higher in diabetic patients, but DBP was similar to that in healthy 
control subjects, although the diabetic patients were receiving anti-hypertensive drugs. The results suggest that diabetic patients do not reach their target BP levels, which represents a cardiovascular risk factor in this group of patients. In addition, the higher SBP and slightly lower DPB are suggestive of arterial stiffness, as manifested by increased pulse pressure, in diabetic patients. Of interest, the plasma PRDX2 and - 6 levels were significantly and negatively correlated with DPB, but not with SBP. This can be explained by a small population size and/or the various levels of hypertensive control in these patients. Therefore, future studies should include larger populations in which patients can be divided into subgroups according to their $\mathrm{BP}$ percentile relative to their PRDX levels. Oxidative stress is consistently observed in hypertension and ROS enhance the development of endothelial dysfunction in hypertension. The relationship between oxidative stress and hypertension has been extensively reviewed elsewhere (17).

Routine markers of inflammation, including the erythrocyte sedimentation rate and CRP, were consistently and positively associated with PRDX1. These findings might reflect a pathophysiologic role of PRDX1 in inflammation. Beyond the traditional cardiovascular risk factors of inflammation, CRP is a crucial factor in the development of atherosclerosis from endothelial dysfunction to plaque rupture and thrombosis. The 2012 Emerging Risk Factors Collaboration Coordinating Center report, which included an extensive population, revealed that the assessment of CRP or fibrinogen levels in people at intermediate risk for a cardiovascular event can help prevent one additional event (18). In addition, other studies have suggested that CRP could be measured as a marker of cardiovascular risk (19) and CRP was a novel marker included in a cardiovascular health study (20). However, it was reported that CRP is associated with oxidative stress in non-diabetic postmenopausal women (21). These findings provide evidence supporting the use of CRP as a marker of cardiovascular disease. We recently reported that PRDX1 levels are correlated with the ankle/brachial index, a clinical marker of peripheral atherosclerosis, in diabetic patients diagnosed with peripheral atherosclerosis (10). Considering these findings, the correlations between PRDX1 levels and markers of cardiovascular risk suggest its levels could contribute to the

\section{References}

1. Antoniades C, Antonopoulos AS, Bendall JK, Channon KM. Targeting redox signaling in the vascular wall: from basic science to clinical practice. Curr Pharm Des 2009; 15: 329342, doi: $10.2174 / 138161209787354230$.

2. Giustarini D, Dalle-Donne I, Tsikas D, Rossi R. Oxidative stress and human diseases: Origin, link, measurement, mechanisms, and biomarkers. Crit Rev Clin Lab Sci 2009; 46: 241-281, doi: 10.3109/10408360903142326.

3. Festa A, D'Agostino R Jr, Howard G, Mykkanen L, Tracy prediction of disease risk and progression, a possibility that warrants further studies. No correlation was found between PRDX4 levels and CRP, although PRDX4 was reported to be a marker of inflammation and oxidative stress in septic patients (22). The lack of a correlation in our study could be explained by the small population size.

Hyperlipidemia and elevated TG levels are recognized cardiovascular risk factors. Of interest, PRDX1 was positively correlated with HDL in the current study. Consistent with our findings, it was reported that PRDX1 helps to prevent excessive endothelial activation and early atherosclerosis (23). We also found that PRDX4 was significantly and negatively correlated with TG levels. Our results are in accordance with a recent report from the American Heart Association that showed that PRDX4, a circulating biomarker with antioxidant properties, was associated with several common cardiovascular risk factors, and was a risk factor for incident cardiovascular disease and all-cause mortality (24).

There are two forms of PRDX1; intracellular PRDX1 is a peroxidase and chaperone protein $(5,25)$ whereas extracellular PDRX1 is a Toll-like receptor 4 (TLR4) ligand (26). Binding of extracellular PDRX1 to TLR4 stimulates the release of the pro-inflammatory cytokines interleukin (IL)-6 and tumor necrosis factor (TNF)- $\alpha$ from macrophages (27) and endothelial cells (26). These unique actions of PRDX1 may explain its multiple associations with cardiovascular risk factors in the current study.

Future studies involving larger populations of prediabetic and diabetic patients are needed to identify the predictive value of PRDX levels in patients at high cardiovascular risk. The differential associations of PRDX1, -2, -4, and -6 with cardiovascular risk factors in our study might suggest different mechanisms and roles in the management of cardiovascular disease risk.

\section{Acknowledgments}

The authors thank the Medical Research Center at King Khalid University Hospital and Medical College, Deanship of Scientific Research at King Saud University for funding and supporting this project. The authors also thank Mr. James Chu for laboratory and technical support.

RP, Haffner SM. Chronic subclinical inflammation as part of the insulin resistance syndrome: the Insulin Resistance Atherosclerosis Study (IRAS). Circulation 2000; 102: 42-47, doi: 10.1161/01.CIR.102.1.42.

4. Ryden I, Grant PJ, Anker SD, Berne C, Cosentino F, Danchin $\mathrm{N}$, et al. ESC guidelines on diabetics, pre-diabetics, and cardiovascular diseases developed in collaboration with the EASD: the task force on diabetes, pre-diabetes, and cardiovascular diseases of the European Society of Cardiology 
(ESC) and developed in collaboration with the European Association for the Study of Diabetes (ESAD). Eur Heart $J$ 2013; 34: 3035-3087, doi: 10.1093/eurheartj/eht108.

5. Rhee SG, Chae HZ, Kim K. Peroxiredoxins: a historical overview and speculative preview of novel mechanisms and emerging concepts in cell signaling. Free Radic Biol Med 2005; 38: 1543-1552, doi: 10.1016/j.freeradbiomed.2005.02.026.

6. Jang HH, Lee KO, Chi YH, Jung BG, Park SK, Park JH, et al. Two enzymes in one; two yeast peroxiredoxins display oxidative stress-dependent switching from a peroxidase to a molecular chaperone function. Cell 2004; 117: 625-635, doi: 10.1016/j.cell.2004.05.002.

7. Memisogullari R, Taysi S, Bakan E, Capoglu I. Antioxidant status and lipid peroxidation in type II diabetes mellitus. Cell Biochem Funct 2003; 21: 291-296, doi: 10.1002/cbf.1025.

8. Bhatia S, Shukla R, Venkata Madhu S, Kaur GJ, Madhava Prabhu K. Antioxidant status, lipid peroxidation and nitric oxide end products in patients of type 2 diabetes mellitus with nephropathy. Clin Biochem 2003; 36: 557-562, doi: 10.1016/S0009-9120(03)00094-8.

9. Kasznicki J, Kosmalski M, Sliwinska A, Mrowicka M, Stanczyk M, Majsterek I, et al. Evaluation of oxidative stress markers in pathogenesis of diabetic neuropathy. Mol Biol Rep 2012; 39: 8669-8678, doi: 10.1007/s11033-012-1722-9.

10. El Eter E, Al Masri A, Habib S, Al Zamil H, Al Hersi A, Al Hussein $F$, et al. Novel links among peroxiredoxins, endothelial dysfunction, and severity of atherosclerosis in type 2 diabetic patients with peripheral atherosclerotic disease. Cell Stress Chaperones 2014; 19: 173-181, doi: 10.1007/s12192013-0442-y.

11. Al-Masri AA, El Eter E, Tayel S, Zamil H. Differential associations of circulating peroxiredoxins levels with indicators of glycemic control in type 2 diabetes mellitus. Eur Rev Med Pharmacol Sci 2014; 18: 710-716.

12. Brinkmann $C$, Neumann E, Blossfeld J, Frese S, Orthmann $\mathrm{P}$, Montiel G, et al. Influence of glycemic status and physical fitness on oxidative stress and the peroxiredoxin system in the erythrocytes of non-insulin-dependent type 2 diabetic men. Exp Clin Endocrinol Diabetes 2011; 119: 559-564, doi: 10.1055/s-0031-1279712.

13. Savu O, lonescu-Tirgoviste C, Atanasiu V, Gaman L, Papacocea R, Stoian I. Increase in total antioxidant capacity of plasma despite high levels of oxidative stress in uncomplicated type 2 diabetes mellitus. J Int Med Res 2012; 40: 709716, doi: 10.1177/147323001204000235.

14. Mehler PS, Coll JR, Estacio R, Esler A, Schrier RW, Hiatt WR. Intensive blood pressure control reduces the risk of cardiovascular events in patients with peripheral arterial disease and type 2 diabetes. Circulation 2003; 107: 753-756, doi: 10.1161/01.CIR.0000049640.46039.52.

15. Estacio RO, Jeffers BW, Gifford N, Schrier RW. Effect of blood pressure control on diabetic microvascular complications in patients with hypertension and type 2 diabetes. Diabetes Care 2000; 23 (Suppl 2): B54-B64.
16. Anderson RJ, Bahn GD, Moritz TE, Kaufman D, Abraira C Duckworth W. Blood pressure and cardiovascular disease risk in the Veterans Affairs Diabetes Trial. Diabetes Care 2011; 34: 34-38, doi: 10.2337/dc10-1420.

17. Schulz E, Gori T, Munzel T. Oxidative stress and endothelial dysfunction in hypertension. Hypertens Res 2011; 34: 665673, doi: 10.1038/hr.2011.39.

18. Kaptoge S, Di Angelantonio E, Pennells L, Wood AM, White IR, Gao P, et al. C-reactive protein, fibrinogen, and cardiovascular disease prediction. N Engl J Med 2012; 367: 13101320, doi: 10.1056/NEJMoa1107477.

19. Greenland P, Alpert JS, Beller GA, Benjamin EJ, Budoff MJ, Fayad ZA, et al. 2010 ACCF/AHA guideline for assessment of cardiovascular risk in asymptomatic adults: a report of the American College of Cardiology Foundation/American Heart Association Task Force on Practice Guidelines. J Am Coll Cardiol 2010; 56: e50-103, doi: 10.1016/j.jacc.2010.09. 001.

20. Shlipak MG, Fried LF, Cushman M, Manolio TA, Peterson D, Stehman-Breen C, et al. Cardiovascular mortality risk in chronic kidney disease: comparison of traditional and novel risk factors. JAMA 2005; 293: 1737-1745, doi: 10.1001/jama 293.14.1737.

21. Park S, Kim M, Paik JK, Jang YJ, Lee SH, Lee JH. Oxidative stress is associated with C-reactive protein in nondiabetic postmenopausal women, independent of obesity and insulin resistance. Clin Endocrinol 2013; 79: 65-70, doi: 10.1111/ j.1365-2265.2012.04512.x.

22. Schulte J, Struck J, Kohrle J, Muller B. Circulating levels of peroxiredoxin 4 as a novel biomarker of oxidative stress in patients with sepsis. Shock 2011; 35: 460-465, doi: 10.1097/SHK.0b013e3182115f40.

23. Kisucka J, Chauhan AK, Patten IS, Yesilaltay A, Neumann C, Van Etten RA, et al. Peroxiredoxin1 prevents excessive endothelial activation and early atherosclerosis. Circ Res 2008; 103: 598-605, doi: 10.1161/CIRCRESAHA.108.174870.

24. Abbasi A, Corpeleijn E, Postmus D, Gansevoort RT, de Jong PE, Gans RO, et al. Peroxiredoxin 4, a novel circulating biomarker for oxidative stress and the risk of incident cardiovascular disease and all-cause mortality. J Am Heart Assoc 2012; 1: e002956, doi: 10.1161/JAHA.112.002956.

25. Chang JW, Lee SH, Lu Y, Yoo YJ. Transforming growth factor-beta1 induces the non-classical secretion of peroxiredoxin-I in A549 cells. Biochem Biophys Res Commun 2006; 345: 118-123, doi: 10.1016/j.bbrc.2006.04.073.

26. Riddell JR, Bshara W, Moser MT, Spernyak JA, Foster BA, Gollnick SO. Peroxiredoxin 1 controls prostate cancer growth through Toll-like receptor 4-dependent regulation of tumor vasculature. Cancer Res 2011; 71: 1637-1646, doi: 10.1158/ 0008-5472.CAN-10-3674.

27. Riddell JR, Wang $X Y$, Minderman $H$, Gollnick SO Peroxiredoxin 1 stimulates secretion of proinflammatory cytokines by binding to TLR4. J Immunol 2010; 184: 10221030, doi: 10.4049/jimmunol.0901945. 